\title{
The Influence of Jet Geometry on the Statistics of Beamed Objects and its Implication for VHE Surveys
}

\author{
G. Henri * \\ UJF-Grenoble 1 / CNRS-INSU, Institut de Planétologie et d'Astrophysique de Grenoble (IPAG) \\ UMR 5274, Grenoble, F-38041, France \\ E-mail: Gilles.Henridobs.ujf-grenoble.fr
}

\section{P.O. Petrucci}

UJF-Grenoble 1 / CNRS-INSU, Institut de Planétologie et d'Astrophysique de Grenoble (IPAG)

UMR 5274, Grenoble, F-38041, France

E-mail: Pierre-Olivier.Petrucci@obs.ujf-grenoble.fr

\section{T. Boutelier}

UJF-Grenoble 1 / CNRS-INSU, Institut de Planétologie et d'Astrophysique de Grenoble (IPAG) UMR 5274, Grenoble, F-38041, France

E-mail: tboutelier@gmail.com

\begin{abstract}
We investigate the role of a finite opening angle on the appearance of relativistic jets. We show that both apparent Doppler factors and superluminal motion are strongly affected by this effect. We simulate several configurations with various dependencies of the Lorentz factor $\gamma_{b}$ as a function of the direction. For each model, the appearance of a moving shell is computed, taking into account relativistic motion and light travel time delays. The apparent velocity is computed by assuming that it is dominated by the brightest point in the sky, corresponding to the maximal possible Doppler factor. It is shown that the apparent velocity is strongly dependent on the smoothness of the variation of $\gamma_{b}$ with the direction. A smooth variation limits the apparent velocity to the inverse of the typical angular range of variation, even for high Lorentz factors. The maximal Doppler factor depends on the precise distribution of $\gamma_{b}$. We discuss the implication of such results on the statistics of beamed sources and the possible luminosity function of CTA extragalactic sources.
\end{abstract}

AGN Physics in the CTA Era - AGN2011,

May 16-17, 2011

Toulouse, France

\footnotetext{
*Speaker.
} 


\section{Introduction}

High energy emission of radio-loud AGNs is thought to arise from a relativistic jet emitted in the vicinity of a central supermassive black hole. The brightest objects, the so-called blazars, are explained by a small angle of this jet with respect to the line of sight (LOS), which amplifies the non-thermal emission thanks to a high Doppler factor. The most simple assumption is the socalled one-zone synchrotron-self Compton (SSC) model. It assumes a spherical zone, in which high energy particles are injected and cooled via synchrotron and Inverse Compton processes. The model depends on the blob radius, the magnetic field, the density and the distribution of the particles, and the relativistic Doppler boosting factor, depending on the velocity and the angle to the LOS, $\delta=1 / \Gamma(1-\beta \mu)$, where $\beta=v / c$ and $\Gamma=\left(1-\beta^{2}\right)^{-1 / 2}$ are respectively the reduced velocity and the bulk Lorentz factor of the jet, and $\mu$ is the cosine of the angle between the velocity and the LOS. In principle, this model can also make predictions on the observed luminosity function of beamed objects and can be compared with so-called "unification" models, comparing luminosities and spectral shapes of beamed vs unbeamed counterparts.

However jet imaging reveals more complex structures than simple blobs, and finite opening angles are observed in different types of objects like AGNs or Young Stellar Objects (e.g. Horiuchi et al. (2006)). Furthermore, there seem to be strong discrepancies between the high Lorentz factors deduced from radiative models, the weak or absent superluminal motions detected in $\mathrm{TeV}$ objects, and the moderate beaming factors ( 3 to 5 ) implied by unification models. Given the strong dependance of the Doppler beaming factor on the angle between the line of sight and the direction of displacement of the emitting region, the jet opening angle should have important effects on the observed jet emission, and the statistics on beamed objects. Gopal-Krishna et al. (2007, and reference therein) have already investigated these effects on the derived geometrical and kinematical parameters of blazar jets like the jet orientation angle, its apparent speed and Doppler factor. However their study didn't include the relativistic aberration caused by light travel effects. We propose a re-analysis of this effect taking properly into account the distorsion of the surface and deduce effective Doppler factors and apparent velocities for open jets. Implications on the statistics of beamed objects will be discussed.

\section{The model}

We consider in the following work, the simple case of a initially spherical shell, propagating with a relativistic speed characterized by the Lorentz factor $\gamma_{0}$ on the jet axis. In the jet rest frame, we assume that the surface emissivity is uniform, its spectrum being described by a power-law of index $n$ defined by $S_{v} \propto v^{-n}$. The geometrical collimation of the jet is characterized by a parameter $\theta_{j}$, and the velocity distribution is described by a function $\gamma(\theta)$, where $\theta$ is the angle to the jet axis. At a point of this surface corresponding to a polar angle $\theta$, the velocity vector is assumed to be parallel to the radial vector.

Four different velocity profiles have been used to study the influence of the jet structure on the appearance of the jet. The first one $\left(D_{1}\right)$ considers the simple case of a conical surface with a constant Lorentz factor $\gamma_{0}$ inside a cone of half-opening angle $\theta_{j}$, and a Lorentz factor equal to unity 
outside (i.e. zero velocity). This distribution is obviously not physical due to the sharp discontinuity at the edge of the cone. In the absence of any specific form argued for in the literature, we have also studied different, smoother distributions. The second distribution $\left(D_{2}\right)$ is built using the conical distribution $D_{1}$, and adding a power-law decrease of index -2 for the Lorentz factor outside the cone. The two last velocity distributions are Gaussian $\left(D_{3}\right)$ and Lorentzian $\left(D_{4}\right)$ profiles. Then for every $|\theta|<\pi / 2$ (pointing-jet), we define:

$$
\begin{aligned}
& D_{1}: \gamma(\theta)=\left\{\begin{array}{l}
\gamma_{0} \text { if }|\theta|<\theta_{\mathrm{j}} \\
\text { no jet outside }
\end{array}\right. \\
& D_{2}: \gamma(\theta)=\left\{\begin{array}{l}
\gamma_{0} \quad \text { if }|\theta|<\theta_{\mathrm{j}} \\
1+\left(\gamma_{0}-1\right)\left(\frac{\theta}{\theta_{\mathrm{j}}}\right)^{-2} \text { else }
\end{array}\right. \\
& D_{3}: \gamma(\theta)=1+\left(\gamma_{0}-1\right) \exp \left[-\ln 2\left(\frac{\theta}{\theta_{\mathrm{j}}}\right)^{2}\right] \\
& D_{4}: \gamma(\theta)=1+\frac{\left(\gamma_{0}-1\right)}{1+\left(\frac{\theta}{\theta_{\mathrm{j}}}\right)^{2}}
\end{aligned}
$$

The symmetrical situation holds for $|\theta|>\pi / 2$ (counter-jet): $\gamma(\theta)=\gamma(\theta+\pi)$.

The apparent surface seen by the observer must be computed at a given observer time and not a given rest frame time. Detailed calculations can be found in Boutelier et al. (2011), and examples of resulting apparent shapes are displayed in Fig. 1. The observed flux on the sky plane is related to the intrinsic flux in the source rest frame $S_{v \text {,int }}$ by the Doppler factor: $S_{v, \text { obs }}=S_{v \text {,int }} \delta^{3+n}$, where $n$ is the energy spectral index. Due to the velocity distribution $\gamma(\theta)$, each point shell have an intrinsic velocity different in norm and direction, and then a different apparent speed as measured by the observer.

Considering the way that observers compute the position of superluminal blobs, we will define the apparent Doppler factor and velocity by those attached to the brightest point on the sky. We thus compute numerically the specific intensity at each point on the sky, and find the maximum one. The problem is actually self-similar since the velocity is assumed to be a constant along each direction. The velocity is thus directly linked to the position of the brightest point, for a given observation time.

Figure 2 shows the evolution of the jet apparent velocity and the Doppler factor as a function of the observational angle $\theta_{\mathrm{obs}}$ for the four velocity profiles $D_{1-4}$ defined in Eqs. 2.1-2.4. We assume a geometrical collimation $\theta_{j}=15^{\circ}$ and a Lorentz factor on the jet axis fixed to $\gamma_{0}=10$. As a comparison, we also display the theoretical curves of the apparent velocity and Doppler factor expected in the case of a perfectly collimated jet with the same Lorentz factor.

\subsection{Conical jet with a constant Lorentz factor}

In the case of the conical velocity profile $D_{1}$ (upper-left corner of Fig. 2), the apparent velocity is equal to 0 for $\theta_{\mathrm{obs}} \leq \theta_{j}$, while the Doppler factor is maximal $\left(\delta=2 \gamma_{0}\right)$. For higher observational 

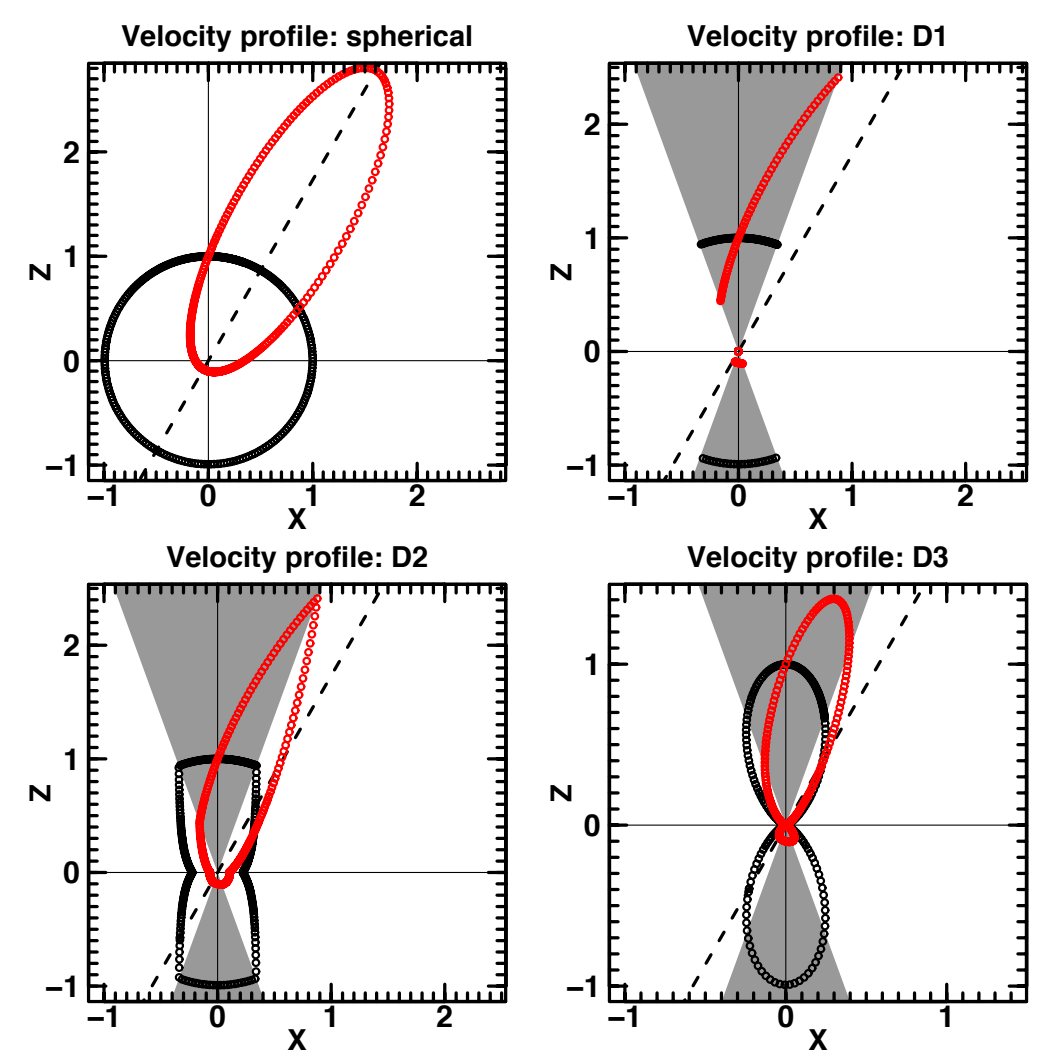

Figure 1: Geometrical surfaces seen by an observer at a given arbitrary observation time $T_{\mathrm{obs}}$, for the non-relativistic case $\left(\gamma_{0}=1.00001\right.$, in black), and for the relativistic case $\left(\gamma_{0}=3\right.$, in red), for a spherical expanding surface (Upper-left case), and the different velocity distributions $D_{1-3}$. The $z$-axis is the symmetry axis of the jet, and $x$ is any orthogonal coordinate. The scales are arbitrary since the problem is self-similar. The shaded region represents the geometrical collimation of the jet $\left(\theta_{j}=20^{\circ}\right)$, and the dashed line is the observer line of sight $\left(\theta_{\mathrm{obs}}=30^{\circ}\right)$.
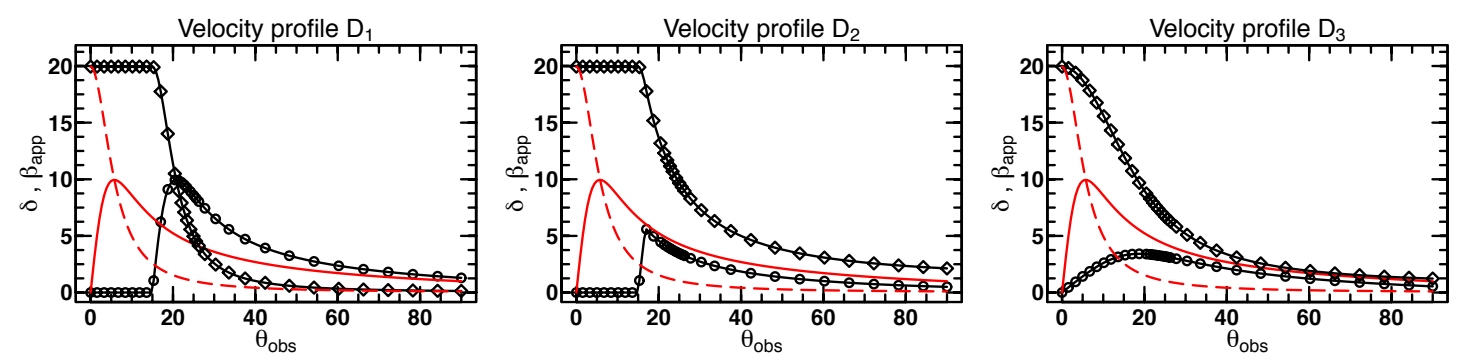

Figure 2: Jet apparent velocity (black empty circles) in unit of $c$ and Doppler factor (black empty diamonds), as a function of the observational angle $\theta_{\mathrm{obs}}$, computed by our model for different jet velocity profiles. Left : velocity distribution $D_{1}$, middle : velocity distribution $D_{2}$, right : velocity distribution $D_{3}$. The jet opening angle is $\theta_{j}=15^{\circ}$, and the Lorentz factor on the jet axis is $\gamma_{0}=10$. For comparison, we have also plotted the theoretical curves for the apparent velocity (red line) and the Doppler factor (red dashed line) of a point source with a Lorenz factor of 10 . 
angles, the apparent velocity and the Doppler factor follow the same behavior as the theoretical expressions, but shifted by an angle $\theta_{j}$.

These results can be easily understood : if the line of sight lies within the jet cone, the maximal intensity will be obtained along the line of sight, at zero angle, where the Doppler factor is maximal. This will always produce a static source with no apparent motion. If the line of sight is out of the cone $\left(\theta_{\mathrm{obs}}>\theta_{j}\right)$, the image is dominated by the emission coming from the edge of the conical jet. Then, the situation becomes similar to a point-like source, seen under the angle $\theta_{\mathrm{obs}}-\theta_{j}$. The evolution of the apparent velocity $\beta_{\mathrm{app}}\left(\theta_{\mathrm{obs}}\right)$ and the Doppler factor $\delta\left(\theta_{\mathrm{obs}}\right)$ is then the same as for the theoretical one in case of a perfectly collimated jet, shifted by $\theta_{j}$.

\subsection{Jet with a continuous velocity profile}

Profile $D_{2}$ is obtained from $D_{1}$ by adding a power-law decrease of the Lorentz factor outside the jet cone (Eq. 2.2), so that the jet velocity smoothly decreases to zero at large $\theta$. The corresponding curves of the shell apparent velocity and Doppler factor as a function of the observational angle (upper-right corner of Fig. 2) are the result of a complicated convolution of the Lorentz factor profile, with the theoretical formula for a point-source. Similar to the $D_{1}$ profile, the apparent velocity is null when the observer looks inside the cone $\left(\theta_{\mathrm{obs}}<\theta_{j}\right)$. As soon as the observational angle is larger than the collimation angle $\left(\theta_{\mathrm{obs}}>\theta_{j}\right)$, the brightest point of the jet moves out from the line of sight, yielding to a non-zero apparent velocity. However, for a given observation angle $\theta_{\text {obs }}$, the apparent velocity is lower than in the case of $D_{1}$. This is due to 2 reasons: 1) the lower intrinsic Lorentz factor $\gamma$ at the position of the brightest point on the shell, compared to the Lorentz factor value $\gamma_{0}$ on the jet axis, and 2) this brightest point is very close to the line of sight with $\alpha<\gamma^{-1}$. this last point is confirmed by the high value of the Doppler factor $\left(\delta>\beta_{\text {app }}\right)$.

Fig. 2 shows the results of the model for a Gaussian velocity profile ( $D_{3}$, Eq. 2.3) The apparent velocity is never null for any observational angle but $\theta_{\mathrm{obs}}=0^{\circ}$, the brightest point of the shell being always slightly shifted out the line of sight. However, we note that the apparent velocity is significantly smaller compared to the point-like source, for the same 2 reasons explained before. As for the $D_{2}$ velocity profile, this is confirmed by the high values of Doppler factor: $\delta>\beta_{\text {app }}, \forall \theta_{\text {obs }}$. We can observe in Fig. 2 that the maximal apparent speed is reached for an observational angle close to the jet opening angle $\theta_{\mathrm{obs}} \approx \theta_{j} \pm \varepsilon$. Similar results are observed for Lorentzian distribution $D_{4}$

\section{Implications on luminosity functions}

All these simulations show that the apparent velocity of a jet is highly dependent on the geometrical collimation and the velocity profile inside the jet. The apparent velocity decreases dramatically as soon as the jet collimation angle doesn't vanish. These results are in agreement with those of Gopal-Krishna et al., although the inclusion of some effects neglected by these authors, such as the time delay between various parts of the shell, and a different prescription for the apparent Doppler factor, modify the precise distribution of apparent Doppler factors as a function of viewing 


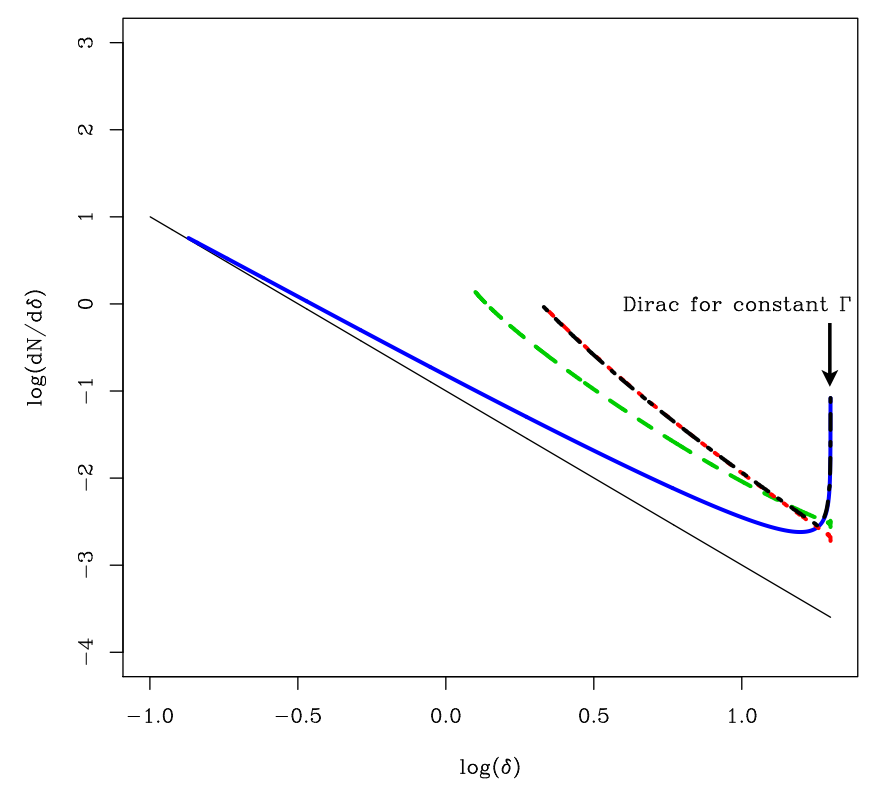

Figure 3: Distribution of Doppler factors deduced from Lorentz factor angular distributions $D_{1-4}$. Solid thin black line : distribution with a single blob; solid blue line : distribution $D_{1}$; dashed black line : distribution $D_{2}$; dashed green line : distribution $D_{3}$; dotted red line : distribution $D_{4}$. The Dirac "spike" is obtained for the distributions $D_{1}$ and $D_{2}$ which include a constant Lorentz factor in a finite solid angle. The distribution $D_{2}$ is essentially the combination of $D_{1}$ inside the cone and $D_{4}$ outside the cone. For $D_{2}$ to $D_{4}$, there is no object with $\delta<1$ since there is a non-relativistic emission at large angle.

angle. This can strongly modify the statistical distribution of apparent velocities and thus the bulk Lorentz factors deduced from these statistical studies.

For a constant intrinsic luminosity, the distribution of Doppler factors will produce a distribution of apparent luminosities, the differential luminosity function being given by

$$
\frac{d N}{d L}=\frac{d N}{d \Omega} \times \frac{d \Omega}{d \delta} \times \frac{d \delta}{d L}
$$

For an isotropic orientation, the first factor is constant. The third one depends on the radiative distribution and the spectral index. Only the second one will depend on the geometrical distribution of Doppler factors, and can be computed from the known distribution $\delta(\theta)$, specifically : $\frac{d \Omega}{d \delta}=2 \pi \sin \theta\left(\frac{d \delta}{d \theta}\right)^{-1}$. The curves corresponding to the various distributions are displayed Fig. 3. For a one-zone, single velocity blob case, the distribution in Lorentz factors is given by $\delta^{-2}$. For other distributions, the slope is different. In the conical case, a constant Lorentz factor $\gamma_{0}$ in a finite angle gives rise to a Dirac-like spike for the maximal possible Doppler factor $2 \gamma_{0}$. This yields a larger number of "bright" objects compared to what would be expected from the simplest model. Note that if the number of beamed objects would be computed from the statistics of beamed (BL Lacs) vs unbeamed (radiogalaxies) objects, like in Henri and Saugé 2006, with an arbitrary threshold, the Lorentz factor deduced would be lower since there is an excess of beamed objects with 
respect to unbeamed ones (a large Lorentz factor produces higher luminosities but fewer objects with a given luminosity). This could be an explanation of the discrepancy between radiative and beaming models.

A realistic luminosity distribution should include an intrinsic luminosity and Lorentz factor distribution. This would be convoluted with the above Doppler distribution and smooth the sharp peaks (including possible Dirac spikes), but for some parameters, a dip in the luminosity distribution could still be obtained. It is thus important to probe the luminosity distribution for fainter objects, and the CTA sensitivity would be an important tool to study this issue.

\section{References}

[1] T. Boutelier, G. Henri, P.-O. Petrucci, The influence of collimation on the appearance of relativistic jets, MNRAS 418 (1913) 2011.

[2] S. Gopal-Krishna Dhurde, P. Sircar, P. J.Wiita, Influence of the jet opening angle on the derived kinematical parameters of blazar jets having uniform and stratified bulk motion, MNRAS 377 (446) 2007.

[3] G. Henri, L. Saugé, The Bulk Lorentz Factor Crisis of TeV Blazars: Evidence for an Inhomogeneous Pileup Energy Distribution?, MNRAS 640 (185) 2006.

[4] S. Horiuchi S., D. L.Meier, R. A. Preston, S. J. Tingay, Ten Milliparsec-Scale Structure of the Nucleus Region in Centaurus A, PASJ 58 (211) 2006 